\title{
Frequency of Confirmed Congenital Renal Malformations in Neonates with Antenatal Diagnosis of Renal Anomalies Presenting In Neonatal Intensive Care Unit of a Tertiary Care Setting of a Low Income Country.
}

\section{Qurat-Ul-Ain Siddique}

National Institute of Child Health

ABID JAMALI ( $\square$ abid.jamali@aku.edu )

Aga Khan University Hospital

\section{Sanober Fatima}

National Institute of Child Health

Jai Parkash

National Institute of Child Health

\section{Research Article}

Keywords: Confirmed congenital renal malformation, antenatal diagnosis, neonatal intensive care unit

Posted Date: August 26th, 2021

DOI: https://doi.org/10.21203/rs.3.rs-831133/v1

License: (c) (1) This work is licensed under a Creative Commons Attribution 4.0 International License. Read Full License 


\section{Abstract}

INTRODUCTION:: Approximately 20 to $30 \%$ of all anomalies identified in the prenatal period are congenital anomalies of the kidney and urinary tract (CAKUT). Widespread antenatal screening has resulted in increased detection of anomalies of the kidneys and urinary tract. There are limited studies on the postnatal confirmation of these antenatally detected renal malformations. Moreover, there is no local data available in this regard. So, we conducted the study so that we can obtain local data as well as we can plan management and prevention protocols for such chronically, and sometimes critically, ill neonates.

MATERIALS AND METHODS:: It was a cross-sectional study conducted at the neonatal intensive care unit (NICU) at the National Institute of Child Health, Karachi during 2017-2018. The sample size was 100 cases. The sampling technique was non-probability consecutive sampling. All neonates aged 1-28 days of either gender admitted in NICU with antenatal diagnosis of congenital renal anomalies on anomaly scan were included in the study. Preterm neonates (gestational age below 34 weeks) and neonates having siblings with similar congenital problems were excluded.

RESULTS:: The mean age of the neonates in our study was $10.17 \pm 9.30$ days and the mean gestational age at birth was $36.65 \pm 1.16$ weeks. The majority of the neonates, that is $65 \%$, were males while $35 \%$ were females. Sixty-six per cent (66\%) neonates were $\leq 10$ days of age while $34 \%$ were $>10$ days of age. Fifty five $\%$ of the neonates were $\leq 36$ weeks of gestation at birth while $45 \%$ were $>36$ weeks of gestation at birth. Frequency of postnatally confirmed congenital renal malformation was observed in $78(78 \%)$ neonates. Neonates whose age at presentation was $>10$ days were slightly more likely to have confirmed congenital renal malformation as compared to neonates with $\leq 10$ days of age, which is $85.3 \%$ vs $74.2 \%$ ( $p$-value 0.206 ). Males and females were found to have almost equal postnatally confirmed congenital renal malformations ( $78.5 \%$ in males and $77.1 \%$ in females) ( $p$-value 0.879 ). Postnatally confirmed congenital renal malformations were observed more commonly in neonates who were $>36$ weeks of gestational age (80\%) as compared to those $\leq 36$ weeks of gestational age (76.4\%) (p-value 0.662$)$.

CONCLUSION::The frequency of confirmed congenital renal malformation was found higher in neonates presenting with antenatal diagnosis of renal anomalies presenting in the neonatal intensive care unit.

\section{Introduction}

Kidney and/or lower urinary tract anomalies that are present at birth in a newborn are generally termed congenital renal malformations. Fetal kidneys can be visualized at 12 weeks, and by 25 weeks, the renal cortex and medulla are distinctly demonstrated on ultrasonography. Approximately 20 to $30 \%$ of all anomalies identified in the prenatal period are congenital anomalies of the kidney and urinary tract (CAKUT). Congenital renal malformations can sometimes present in childhood or adulthood if not picked prenatally or in neonatal age. ${ }^{1}$ 
Widespread antenatal screening has resulted in increased detection of anomalies of the kidneys and urinary tract. ${ }^{2}$ Currently majority of the congenital renal malformations, including CAKUT, are detected antenatally with the use of high sensitivity fetal ultrasonography. ${ }^{3}$ Gestational age based parameters for expected length (appropriate growth) are also available. In the prenatal period, congenital abnormalities of the kidneys and urinary tract (CAKUT) are the most common sonographically identified malformations. Obstructive uropathies account for the majority of cases. ${ }^{4}$

As compared to earlier scanning, mid-trimester ultrasonographic scanning increases the detection rates of congenital renal malformations. Many of these urologic abnormalities would manifest later in life as pyelonephritis, hypertension or end-stage renal disease, if not detected by prenatal ultrasound and subsequently manage. ${ }^{5}$ There are limited studies on the post-natal confirmation of antenatally detected renal malformations. It is suggested that sonographically detected antenatal renal abnormalities should be evaluated by a post-natal sonogram at 4-6 weeks of age and if the 6-week aged scan is normal then further evaluation can safely be limited.

Congenital renal formations are among the most common cause of chronic kidney disease (CKD) in children. Although CKD occurs less often in children than adults, still children account for about $2 \%$ of all patients with ESRD. ${ }^{6}$ Mild forms of congenital kidney disease that appear relatively benign during the childhood may progress to ESRD later in adult life. ${ }^{7}$

Literature has shown that the incidence of confirmed congenital renal malformations is high among neonates who had an antenatal diagnosis of a renal anomaly during the fetal stage (inside the womb). ${ }^{8-}$ ${ }^{10}$ But later study reported the frequency is little lower than previous which showed ambiguity that antenatal diagnosis might have flaws in diagnosis. Moreover, there is no local data available in this regard. So, we conducted the study so that we can gain local data as well as we can plan management and prevention protocols for such critically ill neonates.

\section{Materials And Methods}

It was a cross-sectional study conducted at the neonatal intensive care unit (NICU) at the National Institute of Child Health (NICH), Karachi during 2017-2018. The study was waived for ethical approval from the Ethical Review Committee (ERC) of $\mathrm{NICH}$. The sample size of 100 cases is calculated with $95 \%$ confidence level, $8.5 \%$ margin of error and taking an expected percentage of confirm renal malformation i.e. $77.1 \%$ in neonates presenting with antenatal diagnosis of congenital renal anomalies admitted in NICU. The sampling technique was non-probability consecutive sampling. All neonates aged 1-28 days of either gender admitted in NICU with antenatal diagnosis of congenital renal anomalies on anomaly scan were included in the study. Preterm neonates (gestational age below 34 weeks) and neonates having siblings with similar congenital problems were excluded.

\section{Data Collection Procedure}


Hundred neonates who fulfilled inclusion criteria were enrolled from the NICU of the National Institute of Child Health, Karachi. Informed consent and demographic details (name, age, and gender) were obtained. Then all neonates underwent renal ultrasonography by a single senior radiologist having 4 years of experience. If there was hypoplastic kidney, cystic dysplastic kidney, multicystic dysplastic kidney, aplastic, hydronephrosis or abnormally shaped bladders from midway prenatal compensatory hypertrophy on ultrasound, then congenital renal malformation was confirmed. All this information was recorded on predesigned Performa.

\section{Data Analysis}

All data were entered and analyzed in SPSS version 20.0. The quantitative variables i.e. age and gestational age at birth were presented in the form of mean \pm standard deviation. The qualitative variable i.e. gender and confirm congenital renal malformation was presented in the form of frequency and percentage. Data was stratified for gestational age at birth, age and gender. Chi-square was applied to compare stratified groups taking $p$-value $\leq 0.05$ as significant.

\section{Results}

The mean age of the neonates in our study was $10.17 \pm 9.30$ days and the mean gestational age at birth was $36.65 \pm 1.16$ weeks. Table I. The majority of the neonates, that is $65 \%$, were males while $35 \%$ were females. Sixty-six per cent $(66 \%)$ neonates were $\leq 10$ days of age while $34 \%$ were $>10$ days of age. Regarding the gestational age of the neonates, we found that $55 \%$ of the neonates were $\leq 36$ weeks of gestation at birth while $45 \%$ were $>36$ weeks of gestation at birth. Table II.

The frequency of postnatally confirmed congenital renal malformation was observed in 78 (78\%) neonates. Figure I. Neonates whose age at presentation was $>10$ days were slightly more likely to have confirmed congenital renal malformation as compared to neonates with $\leq 10$ days of age, which is 85.3 $\%$ vs. $74.2 \%$ (p-value 0.206 ). In our study males and females were found to have almost equal postnatally confirmed congenital renal malformations ( $78.5 \%$ in males and $77.1 \%$ in females) (p-value 0.879). Postnatally confirmed congenital renal malformations were observed more commonly in neonates who were $>36$ weeks of gestational age ( $80 \%)$ as compared to those $\leq 36$ weeks of gestational age (76.4\%) (p-value 0.662). Table III

Table I

Mean Age and Gestational Age at Birth of the Patients $(n=100)$.

\begin{tabular}{|llll|}
\hline Variable & Mean \pm SD & Minimum & Maximum \\
\hline age of the patients, days $(n=100)$ & $10.17 \pm 9.30$ & 1 & 28 \\
\hline gestational age at birth, weeks $(n=100)$ & $36.65 \pm 1.16$ & 34 & 38 \\
\hline
\end{tabular}


Table II

Demographic Factors of The Patients $(n=100)$

\begin{tabular}{|lr|}
\hline \multicolumn{2}{|l|}{ GENDER } \\
\hline Male & 65 \\
Females & 35 \\
AGE & \\
$\leq 10$ days & 66 \\
$>10$ days & 34 \\
GESTATIONAL AGE \\
$\leq 36$ weeks & 55 \\
\hline$>36$ weeks & 45 \\
\hline
\end{tabular}

Table III

Comparison of Confirmed Congenital Renal Malformation With Respect to Age, Gender and Gestational Age $(n=100)$

\begin{tabular}{|c|c|c|c|c|c|}
\hline \multirow[t]{2}{*}{ Variables } & & \multicolumn{2}{|c|}{ Confirm Congenital renal malformation } & \multirow[t]{2}{*}{ Total } & \multirow[t]{2}{*}{$\mathrm{p}$-value } \\
\hline & & Yes & No & & \\
\hline \multirow[t]{3}{*}{ Age } & $\leq 10$ days & 49 (74.2) & $17(25.8)$ & $66(100)$ & \multirow[t]{3}{*}{0.206} \\
\hline & $>10$ days & 29 (85.3) & 5 (14.7) & 34 (100) & \\
\hline & Total & $78(78)$ & $22(22)$ & $100(100)$ & \\
\hline \multirow[t]{3}{*}{ Gender } & Male & $51(78.5)$ & $14(21.5)$ & 65 (100) & \multirow[t]{3}{*}{0.879} \\
\hline & Female & $27(77.1)$ & 8 (22.9) & 35 (100) & \\
\hline & Total & $78(78)$ & $22(22)$ & $100(100)$ & \\
\hline \multirow[t]{3}{*}{ gestational age } & $\leq 36$ weeks & $42(76.4)$ & $13(23.6)$ & $55(100)$ & \multirow[t]{3}{*}{0.662} \\
\hline & $>36$ weeks & $36(80)$ & $9(20)$ & $45(100)$ & \\
\hline & Total & $78(78)$ & $22(22)$ & $100(100)$ & \\
\hline
\end{tabular}




\section{Discussion}

Among all sonographically detected antenatal malformations, congenital abnormalities of the kidneys and the urinary tract are the most common. Obstructive uropathies account for the majority of cases. ${ }^{4}$ Renal tract malformations can be familial, and specific mutations of genes involved in renal tract development can sometimes be found in affected individuals. ${ }^{1}$ Widespread antenatal screening has resulted in increased detection of anomalies of the kidneys and urinary tract. ${ }^{2}$

In most countries around the world, it has become a routine to get at least two ultrasonic examinations during pregnancy to monitor fetal development and identify any possible structural malformation. The diagnosis and prognosis of CAKUT have improved significantly in children secondary to gestational ultrasonography. Nowadays $80-90 \%$ of cases of obstructive uropathies can be detected by gestational ultrasonography. The renal malformation detection sensitivity was observed up to $81.2 \%$ in a European study conducted in 12 countries. ${ }^{11}$

Postnatal confirmation of antenatally identified congenital renal malformations was observed in $78 \%$ of neonates. Our data is almost comparable to the study from India (Maharashtra) by Saha et al. where $77.1 \%$ of neonates admitted in NICU with a history of antenatal renal malformations were confirmed postnatally to have renal malformations on ultrasonography. ${ }^{5}$ Although a similar type of study conducted at Jeddah by Bondagji NS concluded that $90.1 \%$ of neonates admitted in NICU with a history of the antenatal renal anomaly on ultrasonography were confirmed to have renal malformations post-natally. ${ }^{12}$

In our study males were predominantly affected as compared to female neonates with a male to female ratio of 1.8:1. Choi H A et al. also reported in their study that males were predominantly affected than females. ${ }^{13}$ Most of the patients presented in NICU before 10th day of life and the mean age at presentation was 10.17 days (range 1-28 days). Gestational age was ranged from 34 to 38 weeks with a mean of 36.65 weeks. Sanghvi et al. also found nearly similar data pretending to the gestational age and they reported the mean 37.1 weeks gestational age at delivery (range 34-40 weeks). ${ }^{14}$

Studies show that the highest yield is obtained if an ultrasonic examination is performed in the third trimester of pregnancy. Positive predictive value (PPV) for APD values $>7 \mathrm{~mm}$ in the third trimester $69 \%$ vs. $49 \%$ for APD $>4 \mathrm{~mm}$ in the second trimester. ${ }^{15}$ Depending on the diagnostic criteria, fetal disease incidence varies from $1-5 \%$ of pregnancies. It is more frequent in boys (3 to $4 / 1)$ predominantly unilateral, and only $20 \%$ of cases will have postnatal clinical significance. Hydronephrosis classification as proposed by the SFU (based on the degree of pelvicalyceal dilation and parenchyma integrity), is another ultrasonographic finding that is important for the diagnosis and prognosis of children with CAKUT. This classification system was actually designed for post-natal scans but studies have shown that if applied properly to ante-natal scans, the children with grades 3 and 4 of hydronephrosis have an increased risk of the need for surgery and renal function deterioration. ${ }^{16}$ 
Certain studies have shown that good gestational ultrasonography not only help in diagnosing congenital renal malformations antenatally but are also very helpful in determining the prognosis of children with CAKUT along with the anticipation of renal function deterioration, like (a) $1 / 3 \mathrm{rd}$ of the cases having single kidney were found to have associated CAKUT, ${ }^{17,18}(\mathrm{~b})$ the probability of CKD at 2 years of age is $15 \%$ when hydronephrosis is associated with other anomalies, as compared to only approximately $2 \%$ with isolated hydronephrosis, ${ }^{19}$ and (c) the risk of renal function deterioration is 9.4-fold higher in cases with bilateral hydronephrosis as compared to unilateral hydronphrosis. ${ }^{20}$

We believe that, even with the methodological limitations of the reviewed studies, the suggestions can help pediatricians to establish clinical hypotheses for the early diagnosis of abnormalities of the genitourinary tract development, by using clinical data and information from the gestational ultrasonography, without resorting to expensive and invasive methods. We believe that the occurrence of any of the clinical signs and symptoms listed here should be considered as an indication to investigate the children regarding the possibility of CAKUT, as there is very limited prospective data to evaluate which of these findings are more important to identify the risk.

\section{Conclusion}

The frequency of confirmed congenital renal malformation was found higher in neonates presenting with antenatal diagnosis of renal anomalies presenting in the neonatal intensive care unit.

\section{Declarations}

\section{Ethics approval}

This study was waived for ethical approval from ethical review committee of National Institute of Child Health Karachi.

\section{Consent for publication}

Informed consent obtained from parents/guardians.

\section{Accordance}

All methods were performed in accordance with the relevant guidelines and regulations

\section{Availability of data and materials}

Most of the data generated or analyzed during this study are included in this article. Limited data can be provided in person on request to the main author. 


\section{Competing interests}

The authors declare that they have no competing interests.

\section{Funding}

This study was not funded by any institution or organization.

\section{Authors' contributions}

QS was the main author who collected data from neonates' parents/guardians and analyzed and interpreted that data. AJ was a major contributor in writing the manuscript along with study design. SF helped in collecting data and interpreting patient's reports and ultrasounds. JP helped in study design and he supervised whole study i.e. from data collection to writing manuscript. All authors read and approved the final manuscript.

\section{Acknowledgements}

Not applicable.

\section{References}

1. Kerecuk L, Schreuder MF, Woolf AS. Renal tract malformations: perspectives for nephrologists. Nat Clin Pract Neph. 2008;4(6):312-25.

2. Sinha A, Bagga A, Krishna A, Bajpai $M$, Srinivas $M$, Uppal R, et al. Revised guidelines on management of antenatal hydronephrosis. Indian pediatrics. 2013;50(2):215-31.

3. Livera LN, Brookfield DS, Egginton JA, Hawnaur JM. Antenatal ultrasonography to detect fetal renal abnormalities: a prospective screening programme. BMJ. 1989;298(6685):1421-1423

4. Hindryckx A, De Catte L. Prenatal diagnosis of congenital renal and urinary tract malformations. Facts, Views \& Vision in ObGyn. 2011;3(3):165-74.

5. Saha A, Batra P, Chaturvedi P, Mehera B, Tayade A. Antenatal detection of renal malformations. Indian Pediatr. 2009;46(4):346.

6. Warady BA, Chadha V. Chronic kidney disease in children: the global perspective. Pediatr Nephrol.2007;22(12):1999-2009

7. Ingelfinger JR, Kalantar-Zadeh K, Schaefer F; World Kidney Day Steering Committee. Averting the legacy of kidney disease: focus on childhood. Kidney Int. 2016;89(6):512-518

8. Gunn TR, Mora JD, Pease P. Antenatal diagnosis of urinary tract abnormalities by ultrasonography after 28 weeks' gestation: incidence and outcome. American journal of obstetrics and gynecology. 
1995 Feb 1;172(2):479 - 86.

9. Hobbins JC, Grannum PA, Berkowitz RL, Silverman R, Mahoney MJ. Ultrasound in the diagnosis of congenital anomalies. American journal of obstetrics and gynecology. 1979 Jun 1;134(3):331 - 45.

10. Zerin JM, Ritchey ML, Chang AC. Incidental vesicoureteral reflux in neonates with antenatally detected hydronephrosis and other renal abnormalities. Radiology. 1993 Apr;187(1):157-60.

11. Wiesel A, Queisser-Luft A, Clementi M, Bianca S, Stoll C, Group ES. Prenatal detection of congenital renal malformations by fetal ultrasonographic examination: an analysis of 709,030 births in 12 European countries. Eur J Med Genet.2005;48:131-44.

12. Bondagji NS. Antenatal diagnosis, prevalence and outcome of congenital anomalies of the kidney and urinary tract in Saudi Arabia. Urol Ann. 2014;6(1):36-40.

13. Choi H, Lee D, Shin S, Lee Y, Ko S, Park S. The Prenatal and Postnatal Incidence of Congenital Anomalies of the Kidneys and Urinary Tract (CAKUT) Detected by Ultrasound. Childhood Kidney Diseases. 2016;20(1):29-32.

14. Sanghvi K, Merchant R, Gondhalekar A, Lulla S, Mehta A, Mehta K. Antenatal diagnosis of congenital renal malformations using ultrasound. Journal of Tropical Pediatrics. 1998;44(4):235-240.

15. Warady B, Chadha V. Chronic kidney disease in children: the global perspective. Pediatr Nephrol. 2007;22:1999-2009.

16. Timberlake MD, Herndon CD. Mild to moderate postnatal hydronephrosis grading systems and management. Nat Rev Urol. 2013;10:649-56.

17. Westland R, Schreuder MF, Ket JC, van Wijk JA. Unilateral renal agenesis: a systematic review on associated anomalies and renal injury. Nephrol Dial Transplant. 2013;28:1844-55.

18. Westland R, Schreuder MF, van Goudoever JB, Sanna-Cherchi S, van Wijk JA. Clinical implications of the solitary functioning kidney. Clin J Am Soc Nephrol. 2014;9:978-86.

19. Quirino IG, Diniz JS, Bouzada MC, Pereira AK, Lopes TJ, Paixão GM, et al. Clinical course of 822 children with prenatally detected nephrouropathies. Clin J Am Soc Nephrol.2012;7:444-51.

20. Quirino IG, Dias CS, Vasconcelos MA, Poggiali IV, Gouvea KC, Pereira AK, et al. A predictive model of chronic kidney disease in patients with congenital anomalies of the kidney and urinary tract. Pediatr Nephrol. 2014;29:2357-64.

\section{Figures}




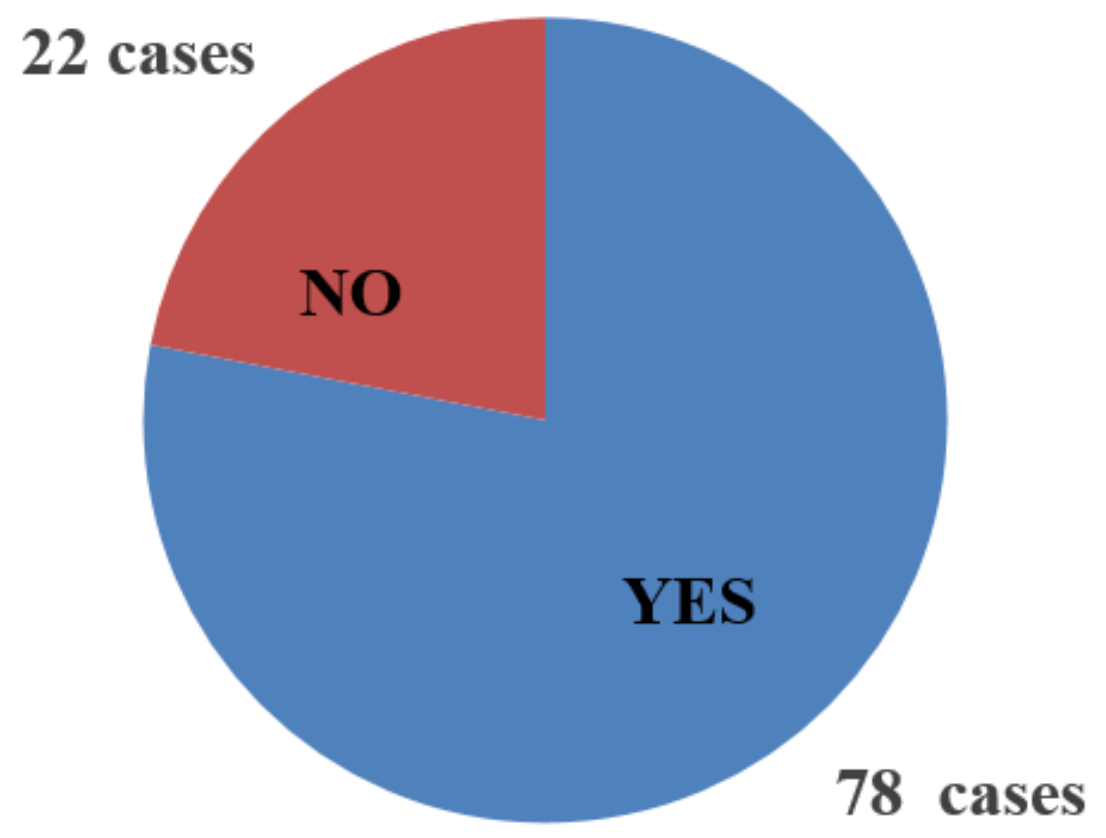

Figure 1

Confirm Congenital Renal Malformation 